\title{
Patterns of Post-Mastectomy Radiotherapy in Immediate Breast Reconstruction - Results from the iBRA-2 Cohort Study
}

Tim Rattay ( $\nabla$ tr104@le.ac.uk)

University of Leicester College of Life Sciences https://orcid.org/0000-0002-2824-4854

Adam Trickey

University of Bristol Medical School

Rachel L O'Connell

Royal Marsden NHS Foundation Trust

Rajiv V Dave

Manchester University NHS Foundation Trust

Joanna Skillman

University Hospitals Coventry and Warwickshire NHS Trust

Nicola LP Barnes

Manchester University NHS Foundation Trust

Matthew D Gardiner

University of Oxford Nuffield Department of Orthopaedics Rheumatology and Musculoskeletal Sciences

Chris Holcombe

Royal Liverpool \& Broadgreen Hospitals NHS Trust

Shelley Potter

University of Bristol

\section{Research Article}

Keywords: Immediate breast reconstruction, breast cancer, radiotherapy, cohort study, trainee collaborative.

Posted Date: December 6th, 2021

DOI: https://doi.org/10.21203/rs.3.rs-1108079/v1

License: (c) (1) This work is licensed under a Creative Commons Attribution 4.0 International License.

Read Full License 


\section{Abstract}

\section{Purpose}

Long-term data indicates that post-mastectomy radiotherapy (PMRT) is associated with improved overall survival in all node-positive breast cancer patients. Immediate breast reconstruction (IBR) remains controversial in the context of planned PMRT, but rates of IBR are increasing. The aim of this study was to examine current practice of PMRT in patients undergoing mastectomy +/- IBR.

\section{Methods}

Data were collected from 2,526 patients enrolled in the iBRA-2 prospective cohort study undergoing mastectomy $+/$ - IBR between $1^{\text {st }}$ July and $31^{\text {st }}$ December 2016 , recruited consecutively at 71 centres across the United Kingdom, Ireland, and five international centres.

Results

Of a total of 2,590 breast procedures included in the analysis, 696 were implant-based, 105 pedicled flap and 230 free-flap reconstruction. $31.4 \%$ of implant-based, $34.3 \%$ of pedicled, and $32.5 \%$ of free-flap reconstructions were recommended for PMRT. PMRT recommendation by cancer stage was $21 \%$ for T1-2 $\mathrm{N} 0,65 \%$ for T1-2 N1, and $89 \%$ for $\mathrm{T}_{\text {any }} \mathrm{N} 2$ and T3 $\mathrm{N}_{\text {any }}$ disease. On multivariate analysis, patients undergoing IBR were no less likely to be recommended for PMRT than patients having mastectomy only. However, patients in Northern Ireland/Ireland, the North of England, and Scotland were less likely to be recommended for PMRT.

\section{Conclusion}

Although IBR was more likely to be performed for lower stage cancers and younger patients with fewer co-morbidities, patients undergoing IBR were no less likely to be recommended for PMRT, irrespective of reconstruction technique. This study also highlighted regional variation in PMRT practice within the UK which merits further investigation.

\section{Introduction}

Breast cancer is the most common female cancer in Europe with 562,000 new cases diagnosed in 2018 [1]. Despite advances in treatment and early diagnosis, almost $40 \%$ of women undergo mastectomy as their primary surgery $[2,3]$. After surgery, radiotherapy is the second most commonly used treatment for breast cancer. Long-term data combining a number of previous randomised-controlled trials demonstrates that post-mastectomy radiotherapy (PMRT) reduces recurrence rate and improves survival in all node-positive breast cancer patients [4]. As a result, the indications for PMRT in women with breast cancer are widening. Population-based data from the US shows that use of PMRT in patients with 1-3 positive lymph nodes increased significantly between 2003 and $2012[5,6]$. Several national treatment 
guidelines now recommend that radiotherapy be considered for all patients with node-positive disease after mastectomy [7-9].

Women undergoing mastectomy are offered immediate breast reconstruction (IBR) with the aim of improving quality of life [10]. IBR remains controversial in the context of planned PMRT [11]. However, an analysis of the US Surveillance, Epidemiology, and End Results (SEER) database indicates that an increase in PMRT amongst women with stage I to III breast cancer undergoing mastectomy did not lead to a concomitant decrease in IBR [6]. In fact, data from the US Mastectomy Reconstruction Outcomes Consortium (MROC) for $>2,000$ patients demonstrates that around $25 \%$ of implant-based and half of autologous reconstructions received PMRT [12]. The UK National Institute of Health and Care Excellence (NICE) recently reviewed 23 observational studies and concluded that there was insufficient evidence to indicate worse patient outcomes from PMRT in the setting of IBR, and that IBR should be offered even if adjuvant RT is anticipated [13].

Currently, high quality evidence for the use of PMRT in intermediate-risk breast cancer (defined as pT12N1; pT3N0; or PT2N0 if also grade III or with lympho-vascular invasion) is lacking and the results of a large European randomized-controlled trial are awaited [14]. At the same time, rates of IBR in the UK, in particular implant-based reconstruction, are increasing [15], yet large scale population-based data on the use of PMRT in patients undergoing IBR has not been published to date. In reconstructive breast surgery, the trainee research collaborative model has recently emerged as a time and cost-effective method for delivering large-scale prospective studies $[16,17]$.

This Breast Reconstruction Research Collaborative of breast and plastic surgeons was utilized to deliver the iBRA-2 study to determine the impact of IBR on the delivery of adjuvant treatment [18]. The present study was undertaken to analyse patterns of PMRT amongst patients undergoing mastectomy with and without IBR enrolled in the iBRA-2 cohort.

\section{Methods}

The methods of the iBRA-2 cohort study have been reported elsewhere $[18,19]$. Briefly, all breast and plastic surgical units performing mastectomy with and without IBR were invited to participate in the study via the professional associations (Association of Breast Surgery [ABS] and British Association of Plastic Reconstructive and Aesthetic Surgeons [BAPRAS] and the breast and plastic surgery collaborative research networks (Reconstructive Surgery Trials Network [RSTN] and the Mammary Fold Academic and Research Collaborative [MFAC]). Of 90 centres, who expressed an initial interest, 76 centres enrolled 2,526 consecutive patients between 1 st July and 31st December 2016. The study sample covered approximately half of all UK breast units as well as five centres in Ireland (recruiting $n=103$ ), four centres in Italy and one in Egypt (recruiting $n=145$ ).

Consecutive women aged 18 or over undergoing mastectomy with or without IBR using any technique for invasive or pre-invasive (ductal carcinoma in situ, DCIS) breast cancer with curative intent were included. Patients were identified from multidisciplinary team (MDT) meetings; unit operating records and clinics. 
Excluded were patients undergoing risk-reducing surgery (without a therapeutic mastectomy for breast cancer), partial mastectomy including wide local excision with volume replacement (latissimus dorsi mini-flaps; lateral intercostal perforator (LICAP) or thoracodorsal artery perforator (TDAP) flaps) or displacement techniques (therapeutic mammaplasty), and those with distant metastatic disease.

The iBRA-2 study was classified as service evaluation according to the NHS Health Research Authority online decision tool http://www.hra-decisiontools.org.uk/research/, so ethical approval was not required. Each participating centre obtained local governance approvals prior to entering patients into the study. Data were collected prospectively, including baseline demographic and operative data. Oncological data and adjuvant treatment recommendations were collected from post-operative MDT meeting records. Pilot data collected at a number of participating sites between 1st May and 30th June 2016 suggested that adjuvant therapy was unlikely to commence earlier than six weeks post-operatively. Data collection in patients not requiring adjuvant treatment therefore continued from the last definitive cancer surgery until six weeks following surgery.

Study data were collected and managed using REDCap (Research Electronic Data Capture) electronic data capture tools hosted at the Kennedy Institute of Rheumatology, University of Oxford [20, 21]. REDCap is a secure, web-based software platform designed to support data capture for research studies, providing 1) an intuitive interface for validated data capture; 2) audit trails for tracking data manipulation and export procedures; 3 ) automated export procedures for seamless data downloads to common statistical packages; and 4) procedures for data integration and interoperability with external sources. For quality assurance purposes, the lead investigator at each site was asked to independently validate 5-10\% of the data. If concordance between the data entered on REDCap and that independently-validated was $<90 \%$, the unit's data were excluded from the analysis, consistent with the QA procedure used in other collaborative projects.

\section{Study definitions and endpoints}

Primary and secondary outcomes in iBRA-2 were selected based on current best practice [22] and national breast cancer guidelines [23]. The endpoint in the present study was use of adjuvant radiotherapy, defined as the recorded post-operative MDT outcome "radiotherapy recommended" or "radiotherapy to be discussed". For analysis purposes, patients in iBRA-2 were categorised into four groups according to the most complex surgical procedure received as: i) mastectomy only (no reconstruction), ii) mastectomy and IBR with implant-only techniques, iii) mastectomy and IBR with pedicled flaps, and iv) mastectomy and IBR with free-flap techniques.

Implant-based procedures included any reconstruction in which only expanders/implants were used to reconstruct the breast, either with or without biological (e.g. acellular dermal matrix) or synthetic (e.g titanium-coated polypropylene) mesh irrespective of whether the implant/expander was placed in a pre or sub-pectoral position. Pedicled-flap procedures included any pedicled-flap used to reconstruct the breast with or without an implant/expander, including latissimus dorsi (LD) and transverse rectus abdominus 
myocutaneous (TRAM) flaps. Free-flap procedures included any technique in which a microvascular freeflap was used for IBR, including deep inferior epigastric perforator (DIEP), superficial inferior epigastric perforator (SIEA), superior and inferior gluteal artery perforator (SGAP and IGAP), and transverse upper gracilis (TUG) flaps. All complications were defined a priori [19]. Major complications were defined as any complication requiring re-admission or re-operation. Minor complications were defined as those managed conservatively. An analysis of short-term complications is described elsewhere [18].

\section{Statistical analysis}

Descriptive summary statistics were calculated for each variable in the whole cohort and sub-divided by procedure type (mastectomy only; implant-based reconstruction; pedicled reconstruction; and free-flap reconstruction). Categorical data were summarised by counts and percentages, and continuous data by median, interquartile range (IQR), and range. Procedure groups were compared using appropriate nonparametric statistics. Univariable and multivariable logistic regression were used to explore associations between clinico-pathological variables and use of PMRT, including patient and procedure-related factors, namely, age, body mass index (BMI), smoking status, American Society of Anaesthesiologists' (ASA) grade; diabetes, ischaemic heart disease (IHD); other comorbidities, chemotherapy, ER- and HER-2 status, lymphovascular invasion, tumour grade, multi-focality, and procedure type.

To assess selection bias, correlation between proportions of patients undergoing PMRT and IBR was determined by region using Pearson's r. Regional trends in PMRT across the United Kingdom and Ireland were further assessed in multivariable logistic regression, adjusted for BMl, co-morbidities, smoking status, ASA, ER- and HER2-status, lymphovascular invasion, multi-focality and tumour grade, and including clustering by centre. All analyses were performed in STATA 15 (STATA Inc, Texas).

\section{Results}

Of 2,526 patients recruited into the iBRA-2 study, 666 underwent implant-based reconstruction, 105 received pedicled flaps, and 227 underwent free-flap reconstruction. The remainder of patients underwent mastectomy only without reconstruction. As 80 patients had bilateral cancer surgery and there were 16 procedures with missing outcome data, a total of 2,590 breast procedures were included in the analysis. On a per breast basis, Table 1 summarises patient demographics and adjuvant therapy decisions by procedure type. Patients undergoing mastectomy alone ( $n=1,559$ breast procedures) were older than those undergoing IBR and were more likely to have co-morbidities as indicated by ASA grade. Patients undergoing IBR were more likely to have lower stage cancers. Adjuvant chemotherapy was more likely to be recommended after mastectomy-only than after IBR. Adjuvant radiotherapy was recommended or for discussion in $44.9 \%$ of mastectomies, $31.4 \%$ of implant-based reconstructions, and in $34.3 \%$ and $32.5 \%$ of pedicled and free-flap reconstructions, respectively $(p<0.001)$. 
Table 1

Patient demographics and proportion of patients offered adjuvant radiotherapy in the iBRA-2 study by type of surgical procedure.

\begin{tabular}{|c|c|c|c|c|c|c|c|c|c|}
\hline \multirow{3}{*}{$\begin{array}{l}\begin{array}{l}\text { Per breast } \\
\text { data }\end{array} \\
\text { Age (median, } \\
\text { IQR) }\end{array}$} & \multirow{2}{*}{\multicolumn{2}{|c|}{$\begin{array}{l}\text { Mastectomy } \\
\text { only }\end{array}$}} & \multirow{2}{*}{\multicolumn{2}{|c|}{$\begin{array}{l}\text { Implant } \\
\text { reconstruction } \\
(n=696)\end{array}$}} & \multirow{2}{*}{\multicolumn{2}{|c|}{ 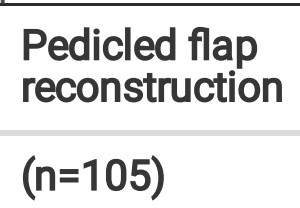 }} & \multirow{2}{*}{\multicolumn{2}{|c|}{$\begin{array}{l}\text { Free flap } \\
\text { reconstruction } \\
(n=230)\end{array}$}} & \multirow{3}{*}{$\begin{array}{l}\begin{array}{l}\text { p- } \\
\text { value }\end{array} \\
<0.001\end{array}$} \\
\hline & & & & & & & & & \\
\hline & 65 & $54-75$ & 50 & $43-57$ & 52 & $47-60$ & 50 & $45-56$ & \\
\hline \multicolumn{10}{|l|}{$\begin{array}{l}\text { ASA grade (n, } \\
\%)\end{array}$} \\
\hline 1 & 333 & $21.4 \%$ & 273 & $39.2 \%$ & 40 & $38.1 \%$ & 59 & $25.7 \%$ & $<0.001$ \\
\hline 2 & 906 & $58.1 \%$ & 379 & $54.5 \%$ & 61 & $58.1 \%$ & 160 & $69.6 \%$ & \\
\hline 3 & 279 & $17.9 \%$ & 23 & $3.3 \%$ & 3 & $2.9 \%$ & 8 & $3.5 \%$ & \\
\hline Missing data & 72 & $4.6 \%$ & 21 & $3.0 \%$ & 1 & $1.0 \%$ & 3 & $1.3 \%$ & \\
\hline \multicolumn{10}{|l|}{ T stage $(\mathrm{n}, \%)$} \\
\hline Tis & 141 & $9.0 \%$ & 161 & $23.1 \%$ & 26 & $24.8 \%$ & 57 & $24.8 \%$ & $<0.001$ \\
\hline T1-2 & 1199 & $76.9 \%$ & 448 & $64.4 \%$ & 64 & $61.0 \%$ & 139 & $76.9 \%$ & \\
\hline T3 & 189 & $12.1 \%$ & 53 & $7.6 \%$ & 9 & $8.6 \%$ & 18 & $12.1 \%$ & \\
\hline Missing data & 30 & $1.9 \%$ & 34 & $4.9 \%$ & 6 & $5.7 \%$ & 16 & $1.9 \%$ & \\
\hline \multicolumn{10}{|l|}{ N stage $(\mathrm{n}, \%)$} \\
\hline NO & 910 & $58.4 \%$ & 524 & $75.3 \%$ & 71 & $67.6 \%$ & 158 & $68.7 \%$ & $<0.001$ \\
\hline N1 & 372 & $23.9 \%$ & 110 & $15.8 \%$ & 16 & $15.2 \%$ & 42 & $18.3 \%$ & \\
\hline N2 & 247 & $15.8 \%$ & 41 & $5.9 \%$ & 12 & $11.4 \%$ & 14 & $6.1 \%$ & \\
\hline Missing data & 30 & $1.9 \%$ & 21 & $3.0 \%$ & 6 & $5.7 \%$ & 16 & $7.0 \%$ & \\
\hline \multicolumn{10}{|l|}{$\begin{array}{l}\text { Chemotherapy } \\
(n, \%)\end{array}$} \\
\hline Recommended & 421 & $27.0 \%$ & 154 & $22.1 \%$ & 28 & $26.7 \%$ & 46 & $20.0 \%$ & 0.001 \\
\hline For discussion & 138 & $8.9 \%$ & 36 & $5.2 \%$ & 5 & $4.8 \%$ & 9 & $3.9 \%$ & \\
\hline $\begin{array}{l}\text { For Oncotype } \\
D^{T X^{T M}}\end{array}$ & 95 & $6.1 \%$ & 64 & $6.1 \%$ & 5 & $4.8 \%$ & 17 & $7.4 \%$ & \\
\hline Missing data & 6 & $0.4 \%$ & 6 & $0.9 \%$ & 0 & $0 \%$ & 0 & $0 \%$ & \\
\hline
\end{tabular}




\begin{tabular}{|llllllllll|}
\hline $\begin{array}{l}\text { Per breast } \\
\text { data }\end{array}$ & \multicolumn{2}{l}{$\begin{array}{l}\text { Mastectomy } \\
\text { only }\end{array}$} & \multicolumn{2}{l}{$\begin{array}{l}\text { Implant } \\
\text { reconstruction }\end{array}$} & \multicolumn{2}{l}{$\begin{array}{l}\text { Pedicled flap } \\
\text { reconstruction }\end{array}$} & \multicolumn{2}{l|}{$\begin{array}{l}\text { Free flap } \\
\text { reconstruction }\end{array}$} & $\begin{array}{l}\text { p- } \\
\text { value }\end{array}$ \\
\hline Recommended & 614 & $39.4 \%$ & 198 & $28.4 \%$ & 35 & $33.3 \%$ & 62 & $27.0 \%$ & $<0.001$ \\
\hline For discussion & 86 & $5.5 \%$ & 19 & $2.7 \%$ & 1 & $1.0 \%$ & 19 & $5.5 \%$ & \\
\hline Missing data & 4 & $0.3 \%$ & 9 & $1.3 \%$ & 0 & $0 \%$ & 0 & $0 \%$ & \\
\hline
\end{tabular}

\section{PMRT recommendation by tumour and nodal stage}

Figure 1 shows the proportion of patients on a per-breast basis $(n=2,590)$ recommended for PMRT by tumour stage. Out of all patients with T3, N2 or N3 stage disease, $89 \%$ were recommended for radiotherapy, while $64.7 \%$ of patients with $\mathrm{N} 1$ stage disease were recommended for radiotherapy. Interestingly, $13.2 \%$ of Tis and $21.1 \%$ of N0 stage patients were also recommended for radiotherapy. When sub-divided by tumour and nodal stage, there were no differences between surgical procedure types (see Supplementary Table).

Table 2 shows the patient and tumour variables associated with PMRT recommendation. In multivariable analysis, patients with larger tumours and more advanced lymph node state were more likely to receive a recommendation for PMRT. However, patients undergoing IBR were as likely as those having simple mastectomy to be recommended for PMRT, although there was evidence of a trend for women receiving implant-based reconstruction to be less likely to be offered PMRT compared to patients undergoing mastectomy only (OR 0.56, $\mathrm{Cl}$ 0.44-0.73, $\mathrm{p}<0.001$ univariable; OR 0.69, 0.45-1.04, $\mathrm{p}=0.08$ multivariable).

Table 2. Predictors of PMRT recommendation. MVA also included age, body mass index, co-morbidities (ischaemic heart disease, diabetes, other), smoking status, chemotherapy recommendation, ASA grade, ER and HER-2 status, lymphovascular invasion, multi-focality, and tumour grade $(\mathrm{Cl}=$ confidence interval). Full results are shown in Supplementary Tables. 


\begin{tabular}{|c|c|c|c|c|c|c|}
\hline \multirow[t]{2}{*}{ Per breast data } & \multicolumn{3}{|c|}{ Univariable analysis } & \multicolumn{3}{|c|}{$\begin{array}{c}\text { Multivariable analysis } \\
(\mathrm{n}=2,000)\end{array}$} \\
\hline & Odds ratio & $95 \% \mathrm{CI}$ & $p$ value & Odds ratio & $95 \% \mathrm{CI}$ & $\mathrm{p}$ value \\
\hline $\begin{array}{l}\text { Tumour size (invasive) } \\
0-9 \mathrm{~mm} \\
10-20 \mathrm{~mm} \\
20-50 \mathrm{~mm} \\
>50 \mathrm{~mm}\end{array}$ & $\begin{array}{r}1 \\
0.74 \\
1.91 \\
14.07\end{array}$ & $\begin{array}{r}\text { Comparator } \\
0.57-0.95 \\
1.48-2.46 \\
8.91-22.24\end{array}$ & $\begin{array}{r}0.02 \\
<0.001 \\
<0.001\end{array}$ & $\begin{array}{r}1 \\
0.75 \\
1.44 \\
18.45\end{array}$ & $\begin{array}{r}\text { Comparator } \\
0.51-1.08 \\
1.01-2.06 \\
8.45-40.26\end{array}$ & $\begin{array}{r}0.12 \\
0.046 \\
<0.001\end{array}$ \\
\hline $\begin{array}{l}\text { Lymph nodes } \\
\text { N0 } \\
\text { N1 } \\
\text { N2 }\end{array}$ & $\begin{array}{r}1 \\
8.02 \\
31.37\end{array}$ & $\begin{array}{r}\text { Comparator } \\
6.10-10.54 \\
19.87-49.50\end{array}$ & $\begin{array}{l}<0.001 \\
<0.001\end{array}$ & $\begin{array}{r}1 \\
4.86 \\
15.54\end{array}$ & $\begin{array}{r}\text { Comparator } \\
3.40-6.96 \\
8.53-28.33\end{array}$ & $\begin{array}{l}<0.001 \\
<0.001\end{array}$ \\
\hline $\begin{array}{l}\text { Type of surgery } \\
\text { Mastecomy only } \\
\text { Implant } \\
\text { Pedicled flap } \\
\text { Free flap }\end{array}$ & $\begin{array}{r}1 \\
0.56 \\
0.61 \\
0.65\end{array}$ & $\begin{array}{r}\text { Comparator } \\
0.44-0.73 \\
0.35-1.08 \\
0.42-1.00\end{array}$ & $\begin{array}{r}<0.001 \\
0.09 \\
0.05\end{array}$ & $\begin{array}{r}1 \\
0.69 \\
0.63 \\
0.63\end{array}$ & $\begin{array}{r}\text { Comparator } \\
0.45-1.04 \\
0.33-1.19 \\
0.30-1.33\end{array}$ & $\begin{array}{l}0.08 \\
0.15 \\
0.23\end{array}$ \\
\hline
\end{tabular}

\section{Impact of PMRT use on IBR}

Excluding patients from five centres outside the United Kingdom and Ireland, Figure 2 shows no clear correlation between the proportion of patients recommended for PMRT and of the proportion receiving IBR across the 14 regions $(r=-0.23, p=0.41)$.

\section{Regional variation in PMRT}

Table 3 shows the proportion of patients recommended for PMRT subdivided by regions, excluding 145 patients from centres outside the UK and Ireland. Complete datasets for multivariable analysis were available for 2,010 breast procedures. Adjusting for patient, tumour and other treatment co-variates, women undergoing mastectomy in Northern Ireland/Ireland (OR 0.37, 0.18-0.76, p=0.007), the North of England $(0.38,0.17-0.83, p=0.016)$, West Midlands $(0.43,0.20-0.92, p=0.029)$, and Scotland $(0.36,0.18$ $0.75, p=0.006$ ) were less likely to be recommended for PMRT than in London and the Southeast. For patients undergoing IBR, the regional numbers were too small to subdivide further by procedure type. Nevertheless, women undergoing IBR in Yorkshire/Humberside were less likely to be recommended for PMRT $(O R 0.25,0.09-0.69, p=0.007)$ than in London and the Southeast.

Table 3. Rates of PMRT by region in patients undergoing mastectomy only and those undergoing IBR. Multivariable analysis (MVA) also included age, body mass index, co-morbidities (ischaemic heart disease, diabetes, other) smoking status, chemotherapy recommendation, ASA grade, ER and HER-2 status, lymphovascular invasion, multi-focality, lymph node status, and tumour size and grade $(\mathrm{Cl}=$ confidence interval, $\mathrm{NI}=$ Northern Ireland). Full results are shown in Supplementary Tables. 


\begin{tabular}{|c|c|c|c|c|c|c|}
\hline \multirow[t]{2}{*}{ Per breast data } & \multicolumn{3}{|c|}{$\begin{array}{l}\text { Mastectomy only } \\
(n=1,184)\end{array}$} & \multicolumn{3}{|c|}{$\begin{array}{l}\text { Breast reconstruction (any) } \\
\qquad(\mathrm{n}=816)\end{array}$} \\
\hline & $\begin{array}{l}\text { Percentage RT } \\
\text { recommended }\end{array}$ & $\begin{array}{l}\text { OR }(95 \% \\
\text { CI) MVA }\end{array}$ & $\begin{array}{l}\text { p value } \\
\text { MVA }\end{array}$ & $\begin{array}{l}\text { Percentage RT } \\
\text { recommended }\end{array}$ & $\begin{array}{c}\text { OR }(95 \% \\
\text { CI) } \\
\text { MVA }\end{array}$ & $\begin{array}{l}\text { p value } \\
\text { MVA }\end{array}$ \\
\hline London/Southeast & $53 \%$ & 1 & & $39 \%$ & 1 & \\
\hline East & $48 \%$ & $\begin{array}{r}0.73(0.35 \\
1.49)\end{array}$ & 0.381 & $39 \%$ & $\begin{array}{c}0.54 \\
(0.20 \\
1.45)\end{array}$ & 0.221 \\
\hline East Midlands & $49 \%$ & $\begin{array}{r}0.85(0.38 \\
1.94)\end{array}$ & 0.704 & $24 \%$ & $\begin{array}{c}0.47 \\
(0.17 \\
1.30)\end{array}$ & 0.145 \\
\hline Yorkshire/Humber & $37 \%$ & $\begin{array}{r}0.50(0.20 \\
1.22)\end{array}$ & 0.129 & $24 \%$ & $\begin{array}{c}0.25 \\
(0.09 \\
0.69)\end{array}$ & 0.007 \\
\hline NI/Ireland & $52 \%$ & $\begin{array}{r}0.37(0.18 \\
0.76)\end{array}$ & 0.007 & $47 \%$ & $\begin{array}{c}1.23 \\
(0.41 \\
3.65)\end{array}$ & 0.708 \\
\hline North & $41 \%$ & $\begin{array}{r}0.38(0.17 \\
0.83)\end{array}$ & 0.016 & $25 \%$ & $\begin{array}{c}0.38 \\
(0.15 \\
0.95)\end{array}$ & 0.038 \\
\hline Scotland & $44 \%$ & $\begin{array}{r}0.36(0.18 \\
0.75)\end{array}$ & 0.006 & $48 \%$ & $\begin{array}{c}1.03 \\
(0.38 \\
2.82)\end{array}$ & 0.952 \\
\hline Wales/Southwest & $47 \%$ & $\begin{array}{r}0.76(0.35 \\
1.67)\end{array}$ & 0.493 & $34 \%$ & $\begin{array}{c}1.43 \\
(0.46 \\
4.47)\end{array}$ & 0.542 \\
\hline West Midlands & $47 \%$ & $\begin{array}{r}0.43(0.20 \\
0.92)\end{array}$ & 0.029 & $37 \%$ & $\begin{array}{r}1.24 \\
(0.29 \\
5.25)\end{array}$ & 0.773 \\
\hline
\end{tabular}

\section{Discussion}

The aim of this study was to analyse patterns of PMRT amongst patients undergoing mastectomy with and without IBR. The short-term outcomes and rates of complications in the iBRA-2 patient cohort have been reported elsewhere [18]. In relation to PMRT, the present study found that between $31.4 \%$ and $34.3 \%$ of women undergoing IBR were recommended for or for discussion of radiotherapy compared to $44.9 \%$ of those receiving simple mastectomy. These figures are in keeping with several previously published observational studies showing that between $29 \%$ and $43 \%$ of patients with IBR are irradiated $[12,17,24$, 25]. Although IBR was more likely to be performed for lower stage cancers and in younger patients with fewer co-morbidities, women undergoing IBR were no less likely to be recommended for radiotherapy after adjusting for patient and tumour variables. This adds to a growing body of evidence that in the setting of PMRT, IBR is becoming an acceptable option for women requiring PMRT [26-28] and that PMRT is only one of many factors in the decision for/against IBR which include consideration of patient 
and oncological factors as well as surgeon and patient preferences. Indeed, it has been shown that breast cancer multi-disciplinary teams (MDTs) cannot predict with certainty whether a patient will require adjuvant PMRT [29].

We found no clear correlation between higher proportions of patients recommended for PMRT and lower proportions of IBR across different UK regions. This suggests that participating centres offered all types of IBR irrespective of whether PMRT was likely to be needed in the adjuvant setting, in keeping with findings from a similar US population-based study [6] and current clinical guidelines [13]. The individual choice of IBR technique is likely to depend on several patient and surgical factors as well as locally available expertise and access to immediate free-flap reconstruction. However, in patients requiring PMRT, implant-only IBR is generally associated with more complications compared to autologous IBR $[12,17]$. Early follow-up data from two prospective cohort studies shows lower satisfaction among patients with irradiated implant-only reconstruction [30,31]. Nevertheless, further work is required to establish the impact of PMRT on the long-term outcomes of IBR to provide high-quality data to inform practice. Initial results from non-randomised studies comparing neoadjuvant versus adjuvant radiotherapy in patients scheduled for IBR are promising and demonstrate the feasibility and safety of this approach [32] but the results of randomized trials will take some years to mature.

Compared to previous population-based analyses showing between $30.3 \%$ and $40.5 \%$ of patients with N1 disease (1-3 positive lymph nodes) receiving PMRT by 2012 [5, 6], in our study, $64.7 \%$ of such patients were recommended for PMRT. This confirms the trend of an increasing number of patients with N1 disease being considered for PMRT and it is in keeping with national clinical guidelines [7-9]. Furthermore, the number of patients in our study with non-invasive cancer receiving post-mastectomy radiotherapy appears relatively high compared to an earlier UK study [33], although our study was not restricted to the breast screening population, and the receipt of radiotherapy following mastectomy $y$ for DCIS may be related to margin status, grade, and presence of micro-invasion.

Subdividing the data by UK regions, we found in our study that patients in Northern Ireland/Ireland, Scotland, the North of England and the West Midlands were significantly less likely to be recommended for PMRT. This highlights some interesting geographical variation, which merits further investigation. A similar US-based study found that density of radiation oncology practices was associated with receipt of PMRT in patients with N1 stage breast cancer [5]. Results of the Selective Use of Post Mastectomy Radiotherapy (SUPREMO) trial are awaited and are likely to reduce the variation in practice of PMRT use in intermediate-risk breast cancer (defined as pT1-2N1; pT3N0; or pT2N0 if also grade III or with lymphovascular invasion) [14].

There is accumulating evidence that individual patient and tumour molecular profiles will be able to predict the response to adjuvant treatment. Several phase III trials are currently underway [34] or have already reported on the effectiveness of the 70- or 21-gene recurrence scores in selecting patients for adjuvant chemotherapy $[35,36]$. Genomic-adjusted radiation dose (GARD) therapy may allow personalisation of radiotherapy on the basis of the biological effect of a given physical dose of radiation, 
calculated using individual tumour genomics, and has been shown to predict time to recurrence and overall survival [37]. In terms of complications from IBR, predictive risk models have been externally validated with receipt of PMRT (or not) as a predictor $[38,39]$. Other risk prediction models for individual radiotherapy side-effects in the breast have been validated [40,41], though none in the setting of IBR. In the future, personalised medicine approaches including the patient germline and tumour genomic profile may aid decision-making around IBR and PMRT.

\section{Strengths and Limitations}

To our knowledge, this is the largest prospective cohort study to date in the UK and Ireland investigating patterns of PMRT in patients undergoing mastectomy with and without IBR, but there are several limitations. Due to its observational design, the study is at risk of bias. Patients were recruited consecutively from participating centres but there were baseline differences between the treatment groups. Although we adjusted for known confounders such as cancer stage, patient age, BMI, smoking status, and ASA grade, we acknowledge that it is not possible to adjust for all potential confounders. Our study included patients from 71 centres in the UK and Ireland as well five centres in Italy in Egypt. It is possible that participating units differed from those not taking part. However, approximately half of all UK breast units were represented in the study.

Due to the short follow-up period (up to initiation of first adjuvant treatment), we were only able to analyse treatment recommendations and were unable to ascertain successful receipt or completion of PMRT in all patients. It was not possible to follow patients up to completion of adjuvant therapy with this surgical trainee collaborative design, but collaborations with oncology trainees should allow us to address this issue in the future. Moreover, a data-linkage study is planned in future to explore oncological outcomes in terms of local recurrence, disease-free and overall survival. While it is not possible to establish causation with this observational study design, the design and conduct of randomized controlled trials in the setting of IBR are perceived as difficult due to patient and surgeon's preference for type and timing of breast reconstruction [25].

\section{Conclusions}

The present study provides evidence that approximately one third of patients undergoing IBR were recommended for PMRT. About two thirds of patients with T1-2 N1 disease were recommended for PMRT, which reflects current clinical treatment guidelines for this patient group. Within the UK and Ireland, the study also highlighted regional variation in PMRT practice which merits further investigation. Although IBR was more likely to be performed for lower stage cancers and in younger patients with fewer comorbidities, patients undergoing IBR were no less likely to be recommended for radiotherapy, irrespective of reconstruction technique. Further work is required to establish the impact of PMRT on the long-term outcomes of different types of IBR to help women and surgeons make more informed decisions about breast reconstruction options. 


\section{Declarations}

\section{Competing interests}

TR is currently a National Institute of Health Research (NIHR) Clinical Lecturer (CL-2017-11-02) and was previously funded by a NIHR Doctoral Research Fellowship (DRF-2014-07-079). AT is funded by a Sir Henry Wellcome Fellowship. SP is an NIHR Clinician Scientist (CS-2016-16-019). This work was undertaken with the support of the NIHR Biomedical Research Centre at University Hospitals Bristol NHS Foundation Trust and the University of Bristol. The views expressed in this publication are those of the authors and not necessarily those of the NHS, the NIHR, or the Department of Health and Social Care.

The other authors have no competing interests to declare.

\section{Data availability statement}

The datasets generated during and/or analysed during the current study are available from the corresponding author on reasonable request.

\section{Authors' contributions}

SP and TR conceived the study design (subgroup analysis); TR contributed to the design, conduct, analysis of the data, interpretation of the results and wrote the first draft of the paper. AT performed the analysis, contributed to data interpretation, and drafted the manuscript. RLOC, TR, RVD, SP contributed to the study design for iBRA-2; MDG and JS contributed to study design and interpretation of the data. All authors read and approved the final manuscript.

Members of the iBRA-2 steering group are (in alphabetical order) Nicola LP Barnes, Jane Blazeby, Elizabeth Conroy, Rajiv V Dave, Matthew D Gardiner, Adrian Harnett, Chris Holcombe, Ciara O'Brien, Rachel O'Connell, Shelley Potter, Tim Rattay, and Joanna Skillman.

Local iBRA-2 investigators (alphabetically by centre) and members of the Breast Reconstruction Research Collaborative are PUBMED citable collaborators in this study and were: Aberdeen Royal Infirmary, NHS Grampian; Alain Curnier, Amir Tadros, Ivan Depasquale, Yazan A Masannat Elizabeth Smyth, Mairi Fuller, Roger Bourne, Steven Heys, Ishrak Hamo. Addenbrookes Hospital, Cambridge Universities NHS Foundation Trust; Fatima Aloraifi, Laura Fopp, Radhika Bali, Sarah Bache, Sarah L Benyon, Michael S Irwin, Amit Agrawal, Charles M Malata. Airedale NHS Foundation Trust; Claire Murphy. Basildon and Thurrock Hospitals NHS Foundation Trust; Adam Misky, Dennis Wayne Chicken. Beaumont Hospital and the RCSI; Nassreen Abdullah, Arnold D K Hill. Belfast City Hospital, Belfast Health and Social Care Trust; Carolyn Cullinane, Gareth Irwin, Stuart A McIntosh, Sigi Refsum, Samantha Sloan, Peter Mallon. Betsi Cadwaladr University Health Board; Chiara Sirianni, llyas Khattak, Chiara Sirianni. Blackpool Teaching Hospitals NHS Foundation Trust; Geerthan Nagachandra, Pasupathy Kiruparan, Debasish Debanth. 
Breast Care Centre, North Bristol NHS Trust, Southmead Hospital, Bristol; Simon Davey, Terry-Ann Curran, Matilda Svenning, Sasirekha Govindarajulu, Zenon Rayter, Rachel Ainsworth, Simon Cawthorn, Ajay Sahu, Sherif Wilson, Elena Prousskaia. Breast Unit Department. University of Naples “Federico II", Naples, Italy; Antonello Accurso, Nicola Rocco, Rosa Di Micco, Antonello Accurso, Gennaro Limite. Breast Unit Department S. Maria delle Grazie ASL Na 2 Pozzuoli; Raffaele Ceccarino, Raffaele Liccardo, Guido Coco. Broomfield Hospital, Mid Essex Hospital Services NHS Trust; Metin Nizamoglu, Mary Morgan, Venkat Ramakrishnan. Cannizzaro Hospital, Catania; Giuseppe Catanuto. Castle Hill Hospital, Hull and East Yorkshire Hospitals NHS Trust; Alex Wilkins, Penelope McManus, Peter Kneeshaw, Kartikae Grover, Tapan Mahapatra, Brendan Wooler, Bilal Elahi, Naila Ihsan. Charing Cross Hospital, Imperial College Healthcare NHS Trust; Alexandra Bucknor, Dimitris Reissis, Judith Hunter, Simon Wood, Navid Jallali, Francis P Henry, Liaquat S Verjee, Jason Lee. Chesterfield Royal Hospital NHS Foundation Trust; Shazia M Khan, Iman Azmy, Julia Massey, Ciaran Hollywood, Michael Oluwajana. Countess of Chester Hospital NHS Foundation Trust; Sonia Bathla, Joanna Seward, Claudia Harding-MacKean. Darent Valley Hospital, Dartford and Gravesham NHS Trust; Risha Lane, Kothandaraman Murali, Bashishta Biswas, Pawel Trapszo, Seema Seetharam. Dorset County Hospital NHS Foundation Trust; Katy Kennedy, Louise Alder, Tomasz Graja. East Cheshire NHS Trust; Khalid Amin, Jalal Kokan, Chandeena Roshanlall. Edinburgh Breast Unit, Western General Hospital, NHS Lothian; Emma Gill, Dhananjay Kulkarni, JM Dixon, Oliver Young, Talha Saleem. Glasgow Royal Infirmary, NHS Greater Glasgow and Clyde; M Biddle, Marie Kearns, Eva Weiler-Mithoff, Ben Chew, Andy Malyon, John Scott, David McGill, lain Mackay. Glenfield Hospital, University Hospitals of Leicester; Salena Bains, Sara Barrows, Tim Rattay, Simon Pilgrim, Sheila Shokuhi, Kelly Lambert, Frances Kenny, Kalliope Valassiadou, Monika Kaushik, Jaroslaw Krupa, Dimitris Dragoumis. Good Hope Hospital, Heart of England NHS Foundation Trust; Quratul ain, Pavlos Lampropoulos, Sarah Moss, Haitham Khalil, Anwar Haq, Balapathiran Balasubramanian. Guy's and St Thomas' NHS Foundation Trust; Petros Charalampoudis, Hisham Hamed, Ashutosh Kothari, Tibor Kovacs, Michael Douek. Harrogate and District NHS Foundation Trust; Iftikhar Mehmood, Biswajit Ray, Matthew Adelekan. Homerton University Hospital NHS Foundation Trust; Laura Humphreys, Salim Tayeh, Christina Choy, Laila Parvanta. Istituto Oncologico Veneto, Padova, Italy; Silvia Michieletto, Tania Saibene. James Paget University Hospitals NHS Foundation Trust; James O'Brien, Sue Down, Sarah Downey, Jerome Pereira. Lincoln County Hospital, United Lincolnshire Hospitals NHS Trust; A S Sami, Anzors Gvaramadze, Jibril A Jibril, Dinesh Thekkinkattil. Llanelli Peony Breast Unit; S Udayasankar, Saira Khawaja, Yousef Shariaha, Simon Holt. Luton and Dunstable University Hospital; Ruth James, Hirah Rizki, Katharine Kirkpatrick, Duraisamy Ravichandran, Deepak Shrestha. Maidstone and Tunbridge Wells NHS Trust; Ellora Barua, Deepika Akolekar. Mid Cheshire Hospitals NHS Foundation Trust; Ahmed Hamad, Eleftheria Kleidi, Susan Hignett, Vanessa Pope, Salma Naseem. Milton Keynes University Hospital NHS Foundation Trust; Jennifer Isherwood, Rachel Soulsby, Amanda Taylor, Kian Chin. Morriston Hospital, Abertawe Bro Morgannwg University Health Board; Dai Nguyen. Musgrove Park Hospital, Taunton and Somerset NHS Foundation Trust; Francesca Guest, Amanda Thorne. Nevill Hall Hospital, Aneurin Bevan University Health Board; Valentina Lefemine. Norfolk and Norwich University Hospitals NHS Foundation Trust; Chris Kirchhoff, Declan C Murphy, Michelle Lo, Ruth Harcourt, Simon J Pain, Maged I Hussien, Katalin Zechmeister, E.M. Sassoon, Andrea Figus, Richard M Haywood, Rozina Ali, Susanna Alexander, 
Adrian Harnett, Konstantinos Geropantas, Daniel Epurescu. North Middlesex University Hospital; Rebecca Lewis, Oladapo Fafemi, Jasdeep Gahir, Tasha Gandamihardja. Nottingham Breast Institute, Nottingham University Hospitals NHS Trust; Jennett Kelsall, Nazli Muhibullah, Charlene Otieno, Fayyaz Mazari, Marta Dauria, Lisa Whisker, Douglas Macmillan, Eleanor Gutteridge, Tuabin Rasheed, Hazem Khout, Kristjan Asgeirsson, Stephen McCulley. Ospedale Santa Chiara, University of Pisa, Italy; Maria Donatella Mariniello, Manuela Roncella, Matteo Ghilli, Livio Colizzi, Elena Rossetti, Lo Russo Marzia, Loredana Fustaino, Alessandro Quattrini Li. Oxford University Hospitals NHS Foundation Trust; Kate L Harvey, Rebecca Windle, Dionysios Dennis Remoundos, Pankaj Roy, Gael MacLean, Asha Adwani. Peterborough City Hospital, North West Anglia NHS Foundation Trust; Elena Popa, Steven Goh, Geeta Shetty. Poole Hospital NHS Foundation Trust; Sarah Clark. Portsmouth Hospitals NHS Trust; Lorenzo Bernaudo, Avi Agrawal, Lucy Mansfield. Princess Alexandra Hospital NHS Trust; Sally Tebbal, Ashraf Patel, Veronica Grassi. Queen Elizabeth Hospital Birmingham, University Hospitals Birmingham NHS Foundation Trust; Ojas Pujji, Kathryn Hamnett, Naren Basu. Royal Bolton Hospital, Bolton NHS Foundation Trust; Emily Granger, Michael Durbar, Panagiotis Pikoulas, Clare Garnsey, Philip Walker, Angela J Vollermere, loannis Michalakis. Royal Devon and Exeter NHS Foundation Trust; Robin Jones, Mina Youssef, Charlotte Ives, Mohammad Masood, Julie Dunn, Sisse Olsen, Douglas Ferguson, Rachel Tillett. Royal Free London NHS Foundation Trust; Anna Allan, Alex Woollard, Rebecca Canny, Alexander Woollard, Afshin Mosahebi, Stephen Hamilton, Shadi Ghali,Daniel Marsh, Jagdeep Chana, Nilesh Sojitra, Ibby Younis. Royal Hampshire County Hospital, Hampshire Hospitals NHS Foundation Trust; Dick Rainsbury, Natalie Chand, Vasileios Kalles, Anne Stebbing, Kevin Harris, Siobhan Laws. Royal Liverpool and Broadgreen University Hospitals NHS Trust; Chris Holcombe, Anne Tansley, Geraldine Mitchell, Emma de Sousa, Julia Henderson, Mysore Chandrashekar. Royal Marsden NHS Foundation Trust; Bernadette Pereira, Chloe Constantinou, Dalia Elfadl, Foivos Irakleidis, Izaro Hernan, Miriam Byrne, Natalie To, Rachel O'Connell, Jennifer Rusby, Peter Barry, Katerine Krupa, William Allum, Fiona MacNeill, Nicola Roche, Gerald Gui, Kelvin Ramsey, Paul Harris, Stuart James, Kieran Power. Royal United Hospitals Bath NHS Foundation Trust; Shelley Potter, Richard Sutton, Jamie McIntosh, Nicola Laurence. Royal Victoria Infirmary, Newcastle Upon Tyne Hospitals NHS Foundation Trust; Louise MacLennan, Robert Milligan, Henry Cain, Adam Critchley, Joe O'Donoghue, Loraine Kalra, Nick Collis. Salisbury NHS Foundation Trust; Gina Weston-Petrides, Roanne Fiddes, Victoria Brown, Anna Aertssen, Diana Slade-Sharman, Mansoor Khan, Caroline McGuiness. Sant'Andrea Hospital of Rome; Vittoria Amorosi, Santanelli di Pompeo Fabio. St Bartholomew's Hospital, Barts Health NHS Trust; Georgios Exarchos, Natasha Jiwa, Jennifer Hu, Serena Ledwidge, Laura Johnson, Anthony Peel, Naseem Dhooma. St Vincent's University Hospital, Dublin; Eric Farrell, Liam Devane, Ruth Tevlin, Enda McDermott, Ruth Prichard, Denis Evoy, Jane Rothwell, James Geraghty, Colin Morrison, Catriona Lawlor. St. James University Hospital, The Leeds Teaching Hospitals NHS Trust; Fiona Langlands, Lauren Taylor, Philip Turton, Raj Achuthan, Kieran Horgan, Shireen Mckenzie, Brian Hogan, Mark Lansdown, Channegowda Navin. The Ipswich Hospital NHS Trust; Liz Sherwin, Caroline Mortimer, Neeraj Garg. The Mid Yorkshire Hospitals NHS Trust; Rahma Adam, Tahera Arif, Zbigniew Kryjak, Deedar Ali, Ravi Sowdi. The Royal Wolverhampton NHS Trust; Elena Fage, Senthurun Mylvaganam, Pilar Matey, Raghavan Vidya, Tapan Sircar. University Hospital of North Tees; Oubida Asaad, Pud Bhaskar, Matei Dordea. University Hospital of South Manchester; Ada Chrysafi, Damian 
McCartan, Rajiv Dave, Rachel Foster, Rebecca Wilson, Sylvia Okwemba, Yousef Majeed, Ciara O'Brien, Vinod Mathen, John Murphy, Nicola Barnes, Ashu Gandhi, James Harvey, Cliona C Kirwan, Richard Johnson. University Hospitals Coventry \& Warwickshire; Krupali Patel, Maria Dalmau Ribas, Natali Vigneswaran, Tom Challoner, Joanna Skillman, Alan Park, Maged Rizkalla, Abigail Tomlins, Kat McEvoy. University Hospitals of North Midlands NHS Trust; Sadaf Jafferbhoy, Soni Soumian, Sankaran Narayanan, Robert Kirby. West Hertfordshire Hospitals NHS Trust; Sladana Bajrusevic, Joseph Maalo, Michalis Charalambous, Lee Min Lai, Kelvin Chong, Simon Thomson, Sherif Monib. Whiston Hospital, St Helens and Knowsley Teaching Hospitals NHS Trust; Leena Chagla, Riccardo Audisio, Rieka Taghizadeh, Azhar Iqbal. Wirral University Teaching Hospital NHS Foundation Trust; Karen James, Maria Callaghan, Shabbir Poonawala, Jonathan Lund, Raman Vinayagam. Worcestershire Acute Hospitals NHS Trust; Sadaf Jafferbhoy, Steven Thrush, Rachel Bright Thomas, Michelle Mullan, Jevan Taylor. York Teaching Hospital NHS Foundation Trust; Ryo Yoshimura, Tom Mathew, Ben Mancey Jones, Kailas Munot, Rana Nasr, Jenny Piper, Deena El-Sharief. Zagazig University Hospital, Egypt; Mohammed Mustafa.

\$Members of the iBRA-2 Steering Group, the Breast Reconstruction Research Collaborative and Mammary Fold Academic and Research Collaborative are PUBMED citable collaborators and are listed at the end of this manuscript.

TR is an NIHR Clinical Lecturer and SP is an NIHR Clinician Scientist.

Corresponding author: Dr Tim Rattay, Leicester Cancer Research Centre, University of Leicester, Clinical Sciences Building, Leicester LE2 2LX, UK, tr104@le.ac.uk.

Previous communications: Earlier versions of this analysis were presented at the British Association of Surgical Oncology (BASO) Annual Scientific Meeting on $4^{\text {th }}$ November 2018, and at the UK Association of Breast Surgery Conference on $13^{\text {th }}$ May 2019, abstract published in Eur J Surg Oncol (2019) 45 (5): 878. https://doi.org/10.1016/j.ejso.2019.01.190.

\section{References}

1. Ferlay J, Ervik M, Lam F, Colombet M, Mery L, Piñeros M et al (2018) In: Cancer, IAfRo (eds) Global Cancer Observatory: Cancer Today.. In: Lyon, France

2. Garcia-Etienne CA, Tomatis M, Heil J, Friedrichs K, Kreienberg R, Denk A et al (2012) Mastectomy trends for early-stage breast cancer: a report from the EUSOMA multi-institutional European database. Eur J Cancer 48:1947-1956

3. Kummerow KL, Du L, Penson DF, Shyr Y, Hooks MA (2015) Nationwide trends in mastectomy for early-stage breast cancer. JAMA Surg 150:9-16

4. McGale P, Taylor C, Correa C, Cutter D, Duane F, Ewertz M et al (2014) Effect of radiotherapy after mastectomy and axillary surgery on 10-year recurrence and 20-year breast cancer mortality: metaanalysis of individual patient data for 8135 women in 22 randomised trials. Lancet 383:2127-2135 
5. Ohri N, Sittig MP, Tsai CJ, Hwang ES, Mittendorf EA, Shi W et al (2018) Trends and variations in postmastectomy radiation therapy for breast cancer in patients with 1 to 3 positive lymph nodes: $A$ National Cancer Data Base analysis. Cancer 124:482-490

6. Frasier LL, Holden S, Holden T, Schumacher JR, Leverson G, Anderson B et al (2016) Temporal Trends in Postmastectomy Radiation Therapy and Breast Reconstruction Associated With Changes in National Comprehensive Cancer Network Guidelines. JAMA oncology 2:95-101

7. NICE (2018) Early and locally advanced breast cancer: diagnosis and management. National Institute for Health and Clinical Excellence, In: NICE, editor. London

8. Wenz F, Sperk E, Budach W, Dunst J, Feyer P, Fietkau R et al (2014) DEGRO practical guidelines for radiotherapy of breast cancer IV: radiotherapy following mastectomy for invasive breast cancer. Strahlenther Onkol 190:705-714

9. Recht A, Comen EA, Fine RE, Fleming GF, Hardenbergh PH, Ho AY et al (2016) Postmastectomy Radiotherapy: An American Society of Clinical Oncology, American Society for Radiation Oncology, and Society of Surgical Oncology Focused Guideline Update. J Clin Oncol 34:4431-4442

10. Harcourt D, Rumsey N (2001) Psychological aspects of breast reconstruction: a review of the literature. J Adv Nurs 35:477-487

11. Duxbury PJ, Gandhi A, Kirwan CC, Jain Y, Harvey JR (2015) Current attitudes to breast reconstruction surgery for women at risk of post-mastectomy radiotherapy: A survey of UK breast surgeons. Breast (Edinburgh, Scotland) 24:502-512

12. Jagsi R, Momoh AO, Qi J, Hamill JB, Billig J, Kim HM et al (2018) Impact of Radiotherapy on Complications and Patient-Reported Outcomes After Breast Reconstruction.J Natl Cancer Inst. ; 110

13. NICE (2018) [1] Evidence reviews for postmastectomy radiotherapy. Early and locally advanced breast cancer: diagnosis and management. National Institute for Health and Care Excellence, London

14. Kunkler IH, Canney P, van Tienhoven G, Russell NS (2008) Elucidating the role of chest wall irradiation in 'intermediate-risk' breast cancer: the MRC/EORTC SUPREMO trial. Clin Oncol (R Coll Radiol) 20:31-34

15. Mennie JC, Mohanna PN, O'Donoghue JM, Rainsbury R, Cromwell DA (2017) National trends in immediate and delayed post-mastectomy reconstruction procedures in England: A seven-year population-based cohort study. Eur J Surg Oncol 43:52-61

16. O'Connell RL, Baker E, Trickey A, Rattay T, Whisker L, Macmillan RD et al (2018) Current practice and short-term outcomes of therapeutic mammaplasty in the international TeaM multicentre prospective cohort study. Br J Surg 105:1778-1792

17. Potter S, Conroy EJ, Cutress RI, Williamson PR, Whisker L, Thrush S et al (2019) Short-term safety outcomes of mastectomy and immediate implant-based breast reconstruction with and without mesh (iBRA): a multicentre, prospective cohort study. Lancet Oncol 20:254-266

18. O'Connell RL, Rattay T, Dave RV, Trickey A, Skillman J, Barnes NLP et al (2019) The impact of immediate breast reconstruction on the time to delivery of adjuvant therapy: the iBRA-2 study. $\mathrm{Br} J$ Cancer 120:883-895 
19. Dave R, O'Connell R, Rattay T, Tolkien Z, Barnes N, Skillman J et al (2016) The iBRA-2 (immediate breast reconstruction and adjuvant therapy audit) study: protocol for a prospective national multicentre cohort study to evaluate the impact of immediate breast reconstruction on the delivery of adjuvant therapy. BMJ Open 6:e012678

20. Harris PA, Taylor R, Minor BL, Elliott V, Fernandez M, O'Neal L et al (2019) The REDCap consortium: Building an international community of software platform partners. J Biomed Inform 95:103208

21. Harris PA, Taylor R, Thielke R, Payne J, Gonzalez N, Conde JG (2009) Research electronic data capture (REDCap)-a metadata-driven methodology and workflow process for providing translational research informatics support. J Biomed Inform 42:377-381

22. BAPRAS Aa (2012) Oncoplastic breast reconstruction - Guidelines for best practice.

23. NICE (2009) Early and locally advanced breast cancer: diagnosis and treatment [CG80]. National Institute for Health and Care Excellence, London

24. Thomson HJ, Potter S, Greenwood RJ, Bahl A, Barker J, Cawthorn SJ et al (2008) A prospective longitudinal study of cosmetic outcome in immediate latissimus dorsi breast reconstruction and the influence of radiotherapy. Ann Surg Oncol 15:1081-1091

25. Winters ZE, Haviland J, Balta V, Benson J, Reece-Smith A, Betambeau N (2013) Integration of patientreported outcome measures with key clinical outcomes after immediate latissimus dorsi breast reconstruction and adjuvant treatment. Br J Surg 100:240-251

26. Barry M, Kell MR (2011) Radiotherapy and breast reconstruction: a meta-analysis. Breast Cancer Res Treat 127:15-22

27. Schaverien MV, Macmillan RD, McCulley SJ (2013) Is immediate autologous breast reconstruction with postoperative radiotherapy good practice?: a systematic review of the literature. J Plast Reconstr Aesthet Surg 66:1637-1651

28. Ricci JA, Epstein S, Momoh AO, Lin SJ, Singhal D, Lee BT (2017) A meta-analysis of implant-based breast reconstruction and timing of adjuvant radiation therapy. J Surg Res 218:108-116

29. Wilson M, Dordea M, Light A, Serra MP, Aspinall SR (2015) Accuracy of a multidisciplinary team-led discussion in predicting postmastectomy radiotherapy. Ann R Coll Surg Engl 97:198-203

30. Pusic AL, Matros E, Fine N, Buchel E, Gordillo GM, Hamill JB et al (2017) Patient-Reported Outcomes 1 Year After Immediate Breast Reconstruction: Results of the Mastectomy Reconstruction Outcomes Consortium Study. J Clin Oncol 35:2499-2506

31. Sewart E, Turner NL, Conroy EJ, Cutress RI, Skillman J, Whisker L et al (2020) The Impact of Radiotherapy on Patient-reported Outcomes of Immediate Implant-based Breast Reconstruction With and Without Mesh.Ann Surg.

32. Singh P, Hoffman K, Schaverien MV, Krause KJ, Butler C, Smith BD et al (2019) Neoadjuvant Radiotherapy to Facilitate Immediate Breast Reconstruction: A Systematic Review and Current Clinical Trials. Ann Surg Oncol 26:3312-3320

33. Clements K, Dodwell D, Lawrence G, Ball G, Francis A, Pinder S et al (2015) Radiotherapy after mastectomy for screen-detected ductal carcinoma in situ. Eur J Surg Oncol 41:1406-1410 
34. Ramsey SD, Barlow WE, Gonzalez-Angulo AM, Tunis S, Baker L, Crowley J et al (2013) Integrating comparative effectiveness design elements and endpoints into a phase III, randomized clinical trial (SWOG S1007) evaluating oncotypeDX-guided management for women with breast cancer involving lymph nodes. Contemp Clin Trials 34:1-9

35. Cardoso F, van't Veer LJ, Bogaerts J, Slaets L, Viale G, Delaloge S et al (2016) 70-Gene Signature as an Aid to Treatment Decisions in Early-Stage Breast Cancer. N Engl J Med 375:717-729

36. Sparano JA, Gray RJ, Makower DF, Pritchard KI, Albain KS, Hayes DF et al (2018) Adjuvant Chemotherapy Guided by a 21-Gene Expression Assay in Breast Cancer. N Engl J Med 379:111-121

37. Scott JG, Sedor G, Ellsworth P, Scarborough JA, Ahmed KA, Oliver DE et al (2021) Pan-cancer prediction of radiotherapy benefit using genomic-adjusted radiation dose (GARD): a cohort-based pooled analysis. Lancet Oncol.

38. Khavanin N, Qiu CS, Mlodinow AS, Vu MM, Dorfman RG, Fine NA et al (2017) External validation of the breast reconstruction risk assessment calculator. J Plast Reconstr Aesthet Surg 70:876-883

39. Blough JT, Vu MM, Qiu CS, Mlodinow AS, Khavanin N, Fine NA et al (2018) Beyond 30 Days: A Risk Calculator for Longer Term Outcomes of Prosthetic Breast Reconstruction. Plast Reconstr Surg Glob Open 6:e2065

40. Rattay T, Seibold P, Aguado-Barrera ME, Altabas M, Azria D, Barnett GC et al (2020) External Validation of a Predictive Model for Acute Skin Radiation Toxicity in the REQUITE Breast Cohort. Front Oncol 10:575909

41. Kindts I, Defraene G, Petillion S, Janssen H, Van Limbergen E, Depuydt T et al (2019) Validation of a normal tissue complication probability model for late unfavourable aesthetic outcome after breastconserving therapy. Acta Oncol 58:448-455

\section{Figures}




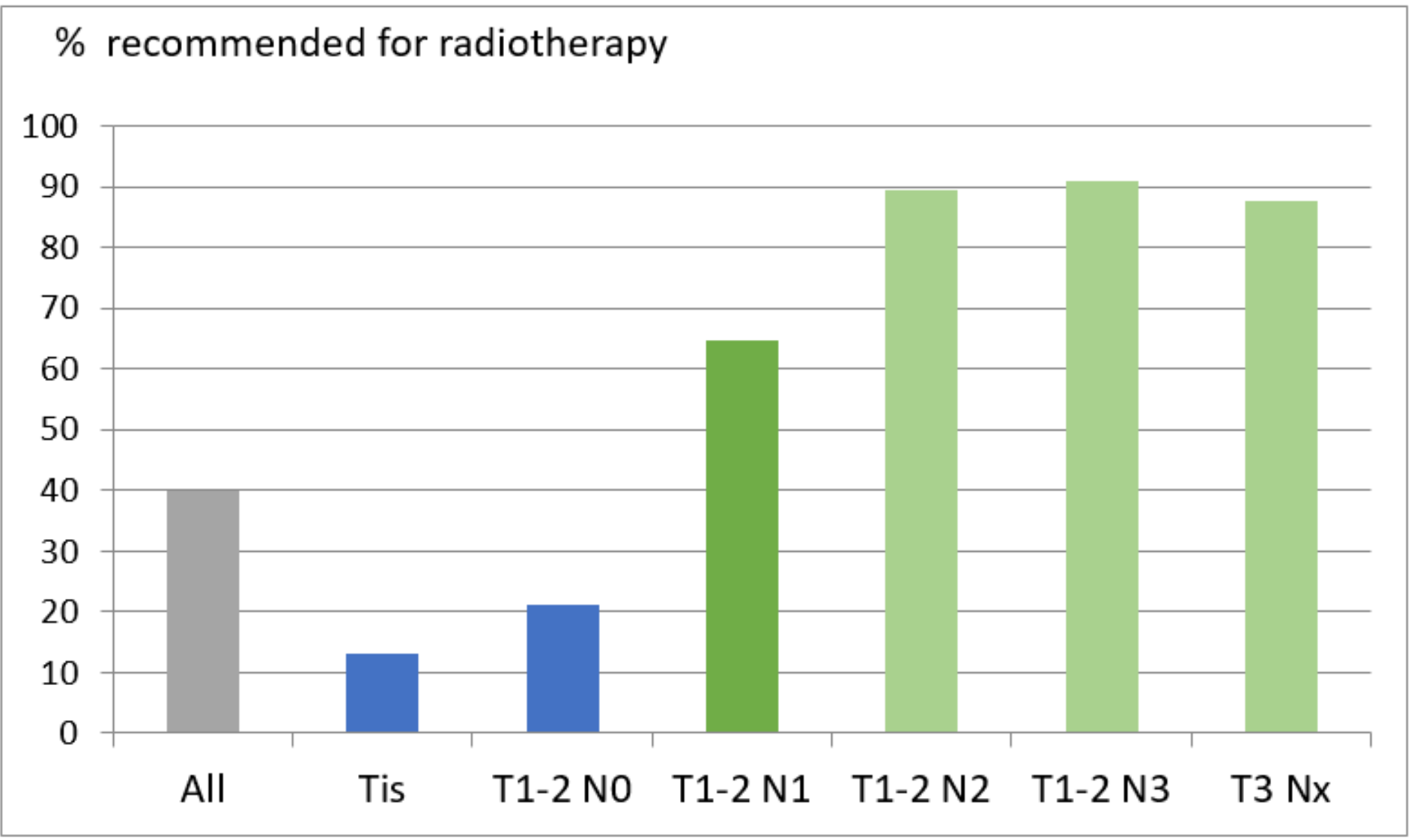

Figure 1

Proportion of patients (\%, per-breast data) in the study recommended for post-mastectomy radiotherapy by tumour stage. 


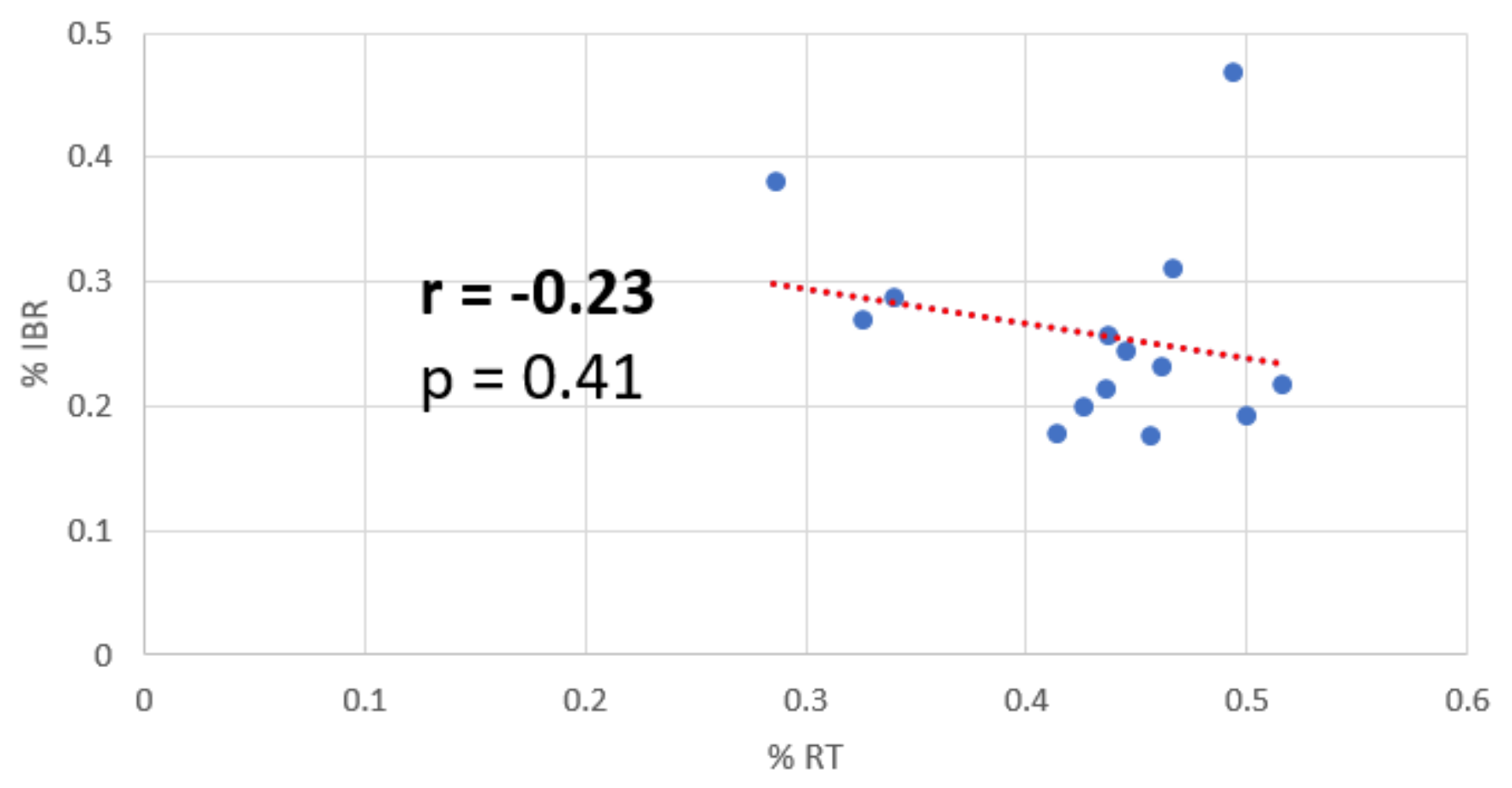

Figure 2

Reconstruction percentages and PMRT recommendation percentages $(n=2,381$, excluding centres outside UK and Ireland).

\section{Supplementary Files}

This is a list of supplementary files associated with this preprint. Click to download.

- iBRA2RadiotherapySupplTables.xlsx 